Original Research Paper

\title{
BOOM: Rainbow's Gravity as the Source of Light Refraction
}

\author{
Mohsen Lutephy \\ Department of Chemistry, Islamic Azad University (IAU), Tehran, Iran
}

\section{Article history}

Received: 16-07-2021

Revised: 30-10-2021

Accepted: 02-12-2021

Email: lutephy@gmail.com

\begin{abstract}
On the fully variable light speed (VSL) universe derived by alliance of Mach inertia principle and Planck's quantized natural units and generalized Minkowski metric, it is extracted the fundamental equation of variable light speed called here Alpha Genesis Prime. Then the fundamental gravity is defined as a vector to justify the light speed variation accordingly. Gravitational vector via a tangential component does set the light speed to be matched with the Alpha Genesis Prime and according to the Pythagorean Theorem; it has also a component normalized to the light velocity. Interestingly by the gravity divided to the tangent and normal components relative to the light velocity we argue the Snell's law of the light refraction and we find that dependency of the refractive index to the volume density is not fundamental but fundamentally the refractive index follows gravitational potential in bound quantum systems. The gravity is enhanced in bound quantum systems extended from Femto-scale (Nucleuses and strong nuclear force), Micro-gravity (fundamental rainbow's gravity), the galaxies and clusters as the large scale bound quantum systems, up to the observable universe which the variable gravitational $G$ is Newtonian (constant $G$ in short cosmic time). The refraction of the light is sourced potentially by Rainbow's gravity in bound quantum systems which the potential integration domain is limited to the wavelength of the photons. Quantum mechanically the photons are force carrier in the range of their wavelength to enhance gravitational $G$ inasmuch as large that atoms to bend the photons similar to enhanced gravity in the nucleuses in the range of hadron wavelengths. Reestablish of the Newtonian mechanics yields to the fundamental gravity which is identical with the refraction of the light and we find that the mechanical potential of the light's refraction is the gravity.
\end{abstract}

Keywords: Quantum Gravity, Cosmology, Planck Units, Planck Stars, Machian Universe, Variable Light Speed, Quantization of the Space-Time

\section{Introduction}

The light refraction is a mystery yet. Newtonian gravity and Einstein general relativity and Maxwell electromagnetism can't answer to the question that why the light is refracted? It is not clear still why the light speed is slowed in the mediums?

We know that the explanations based upon the idea of light scattering from, or being absorbed and re-emitted by atoms are both incorrect. Explanations like these would cause a "blurring" effect in the resulting light, as it would no longer be travelling in just one direction.

As mentioned in the Wikipedia dictionary, ultimate explanation for light refraction has been proposed on the nature of the light as an electromagnetic wave. On this explanation, the light is an oscillating electrical/magnetic wave and the light traveling in a medium causes the electrically charged electrons of the material to also oscillate. Then it is thought that the oscillating electrons emit the electromagnetic waves of its own by this oscillation. It is thought that the emitted electromagnetic waves interact with the incident photons and when the waves interfere in this way, the resulting "combined' wave may have wave packets that pass an observer at a slower rate. The explanation states (Wikipedia) that.

"if it reaches the interface between the materials at an angle one side of the wave will reach the second material first and therefore slow down earlier. With one side of the wave going slower the whole wave will pivot towards that side. This is why a wave will bend away from the surface or toward the normal when going into a slower material. In the opposite case of as wave reaching a material where the speed is higher, one side of the wave will speed up and the wave will pivot away from that side." 
This explanation is unjustified; for imaginary interfere of the incident photons with produced electromagnetic waves by atoms and actually there is no such a chance and there is no any report to reproduce it in the laboratory. Also if the wave packet was the source to decrease the speed of light, then the white light which is also a wave packet had slower speed compared to the monochromatic beams. Also the change of angle is too a big difficulty for this explanation for light refraction. The slowing of a part of the photon which reaches earlier to the different medium has no any mechanical source for change of the photon direction in the interface of the mediums and disintegration of these fictitious wave packets to pure waves, while the waves come out from the medium is also a paradox. Generally this explanation has no any argument for the Snell's law and slowing of the photon speed in the mediums by the group velocity of the wave packets has no any link to the Snell's law. The refractive index is an experimental index and up to now there is no a mechanism to illustrate it and we don't know why the refractive index is varied in different mediums and all are assumptions whereas the Snell's law is very verse and such a law requires a verse origin.

The light is neutral electrically and then electromagnetic force can't refract the light. Also the Fermat least time principle and Huygens-Fresnel principle don't indicate the mechanical source for the light speed variation suppose these principles are technical lemmas.

The argument for the light refraction on the theory of envelope mechanism and group velocity is mistake for many reasons for example the light is refracted when the light is even still not completely interred into the mediums. There should be a mechanical force affecting the photons from side of the atoms. The electromagnetic force can't be the case for the reality that the photons are mainly electrically neutral.

Newtonian gravity is not fundamental because that the newton's gravity is an additional effect of the multi-particle system assumed as a single fundamental mass whereas that additional effect of individual particles may show a different property which is not fundamental but composite mistakenly assumed as a fundamental property. Newton's gravity is approximate. Even here we will show that the fundamental gravity is not centrifugal force but additionally all together in a many-particle static body behaves as a centrifugal resultant force. We show here that the fundamental gravity does work individually for a mass-point particle and then the force on an apple is not fundamental and what we see as a resultant gravity may differ with the fundamental gravity acting on the fundamental particles.

Also the Einstein general relativity isn't a fundamental gravity because that Einstein total field is a proof less formula and consequently modified and many mathematical answers can be obtained by that. The scientists for each puzzle; they try to find a solution from Einstein general relativity to set it with the observations and when they can't find a suitable answer they consider modifications to the general relativity or its solutions. Then they have assumed unobserved mass/energy and undiscovered sub atomic particles for interpretation of observations.

By the way there are many evidences revealing that the Einstein general relativity is not fundamental, for example one of the evidences is that in the Einstein general relativity, it is used a parameter defined as the volume density of the universe whereas that the universe isn't homogenous and principle of cosmology isn't valid. In general relativity, the energy tensor $T_{\mu v}$ is proportional to Riemannian curvature $R_{\mu v}$ but in the vacuum or voids, the energy tensor $T_{\mu \nu}$ is zero when it is defined on the volume density whereas the curvature $R_{\mu v}$ is not zero as a contradiction for GTR. In reality discontinuity of the density is manifest and there is no any volume included to the uniform distribution of the matter inside, whether large scale or small scale. Then every equation included to the volume density is not fundamental for that the volume density is approximate and approximation is not fundamental.

On the Einstein general relativity, the scientists have used the vacuum solution to simulate the solar system whereas in the solar system, the universal potential is large and it is false to simulate the solar system independently which mistakenly assumed in the Schwarzschild (Solution, 1916) for the light passing around the Sun. Also the GTR has answers for vacuum as a manifest contradiction because that the Einstein by Mach inertia principle has claimed that general relativity should be fully on the curvature of space by matter only (Pais, 2005).

On the Mach inertia principle, the mass is not an intrinsic property of the matter until to be defined locally but the mass is an extrinsic property driven by whole of the universe matter in Machian relation that:

$m=m_{0} \frac{G_{N}}{c_{0}^{2}} \sum_{i} \frac{m_{i}}{r_{i}}$

Then the approximate equations defined on volume density should be redefined on the parameter $\sum_{i} m / r$, that is, we need to revise the energy tensor on the parameter $\sum_{i} m / r$.

Einstein total field solutions encounters next paradoxes such as the anisotropy of the light speed by direction, or the existence of the singularities or a region inside the Schwarzschild radius which the scientists have considered it as a black hole. In fact the Einstein general relativity is a continuum frame work mechanics and not possible to apply it precisely in N-body problem properly. Such a continuum mathematical frameworks are indeed two body problems. Even in the Einstein especial relativity, we see that the $\mathrm{N}$-body problem of the Maxwell electromagnetism encounters with relevant paradoxes 
revealing that ultimately the continuum framework can be an approximation for real N-body discrete universe. Then Einstein general relativity is an approximate model, by applying a continuum framework for discrete physics. There are many logical failures in the Einstein general relativity all revealing that the Einstein general relativity is an approximate mathematical model for the gravity.

But the fundamental gravity is agreement with generalized Minkowski metric which the light speed $\mathrm{c}$ is fully variable on the Mach inertia principle. Then the curvature of the space depends exclusively to the light speed and light speed in the universe does determine the curvature fully. Here we find fundamental gravity which reduces to the Newtonian gravity approximately and even mimics GTR. We see that the fundamental gravity and refraction of the light are unified to indicate that the refractive index depends fundamentally to the gravitational potential instead the volume density and we find a true correlation between the refractive index and the parameter $\sum_{i} m_{i} / r_{i}$ in bound quantum systems which we call it here absolute density.

Up to now the idea of scientific consensus is that the light refraction is a local event and in the vacuum the refractive index is at the order of unity however the light speed is variable vacuum to vacuum in different points of the universe. It is believed that the refractive index is an electromagnetic parameter driven via Maxwell electromagnetism. But reestablished Newtonian mechanics yields to the fundamental gravity which is identical with refraction of the light and the mechanical potential of the light refraction is the gravity. We argue here the so called Snell's law of the light refraction by fundamental gravity and we find a precise answer for question why an object is crystal and why other not.

The fundamental gravity is revealing the true rainbow's gravity which is possible to be written in metric format too. The previous rainbow's gravities (Galan and Marugan, 2004; Hackett, 2006; Garattini and Mandanici, 2012; 2014a; Garattini, 2013; Garattini and Majumder, 2014b; Leiva et al., 2009; Li et al., 2009; Ali et al., 2015; Awad et al., 2013; Barrow and Magueijo, 2013; Liu and Zhu, 2008; Ali and Khalil, 2014; Gim and Kim, 2014; Amelino-Camelia et al., 1997; 1998) on the principle of relative locality (Amelino-Camelia et al., 2011) suggest that the gravity affects different wavelengths in the same way that a prism affects the light. The theory was first proposed in 2003 by physicists Lee Smolin and Joao Magueijo and scientists are currently attempting to detect rainbow's gravity using the Large Hadron Collider (Banks and Fischler, 1999; Giddings and Thomas, 2002; Dimopoulos and Landsberg, 2001; Emparan et al., 2000; Meade and Randall, 2008; Antoniadis et al., 1998; da Rocha and Coimbra-Araujo, 2006).
But here we find that the light refraction and the gravity are unified and the electromagnetism isn't the source for the light refraction and derivation of the refractive index by Maxwell electromagnetism is imaginary suppose the photons are electrically neutral and we need to reestablish the physics. Via different phenomenological motivations, a series of Rainbow's Gravity (RG) models have been obtained. Based on the varying speed of light theory, Magueijo and Smolin proposed a kind of Modified Dispersion Relation (MDR) as:

$\frac{E^{2}}{\left(1-\gamma E l_{p}\right)^{2}}-p^{2}=m^{2}$

where, $\gamma, l_{p}$ and $m$ represent the rainbow parameter, the Planck length and the $m$, mass of the test particle. This equation indicates that the space-time has an energy-dependent velocity as:

$c(E)=d E / d p=1-E l_{p}$

Comparing with general form of MDR it has been deduced that:

$E^{2} f^{2}\left(E l_{p}\right)-p^{2} g^{2}\left(E l_{p}\right)=m^{2}$

And from Feng and Yang (2018) we have:

$$
\begin{aligned}
& d s^{2}=-\frac{1-(2 G M / r)}{f^{2}\left(E l_{p}\right)} d t^{2} \\
& +\frac{d r^{2}}{[1-(2 G M / r)] g^{2}\left(E l_{p}\right)}+\frac{r^{2}}{g^{2}\left(E l_{p}\right)} d \Omega^{2}
\end{aligned}
$$

But we indicate here that the light speed doesn't depend to the energy of photon but all photons have equal speeds in the vacuum in each point of the universe and we argue that assumed rainbow's parameter $\gamma$ is zero and still correct the energy-momentum relation $E^{2}-P^{2}=m^{2}$.

The general relativistic rainbow's gravities have no answer for refraction of the light but noticeable for bending of the light around the Schwarzschild radius in modified general relativity whereas we argue here that the fundamental gravity (reestablishment of the Newton's gravity) is the potential source for the light refraction and the atoms bend the light extremely much inasmuch as that the atoms simulate what scientists are thinking to happen around the fictitious black holes. Of course the fundamental gravity does not show any relativistic black hole and ultimately the answer is just spiral geodesy in agreement with experiments performed at LHC with no result for black holes (Chatrchyan et al., 2012a; 2012b). 
We find here also a metric tensor which mimics dependency of the fundamental gravity to the photons wavelength and interestingly we find that some metric tensors in the field of general relativity in extra dimensions mimic the bound quantum systems and general relativity may be generalized to the bound quantum systems like the Newtonian gravity (Lutephy, 2020).

Of course, we don't agree with deformation of the energy-momentum relation applied in relativistic rainbow's gravities. Also we don't agree with extra dimensions applied by rainbow's gravities in the general relativity; however such extra dimensions cause to extend mathematically the solutions as a degree of freedom to embed approximately the bound quantum systems in the general relativity. But a mathematical model does not show ever a true physical mean and we agree the criticism of Sabine Hossenfelder for rainbow's gravities in title "No, the LHC will not make contact with parallel universes" (backreaction.blogspot.com 2015).

Our results here can be modeled approximately via a generalized Schwarzschild metric (1916) which the metric tensor depends to the wavelength of the photons in the way of quantization of boundary in the scale comparable to the wavelength of photons which causes the geodesy of photons to be different by different wavelengths, of course without relation to extra dimensions and parallel universes, verifying the Sabine Hossenfelder statements in her criticism.

We show here that the change of the light speed does relate to the wavelength of the photons for reality that the wavelength of photons does produce quantum mechanically a bound quantum system below the radius equal to the wavelength of the photon and then it is revealed high intensity gravity for limitation of the potential to the boundary of bound quantum systems, exactly similar to the strong gravity observable in the strong nuclear force in which the wavelength of the hadron force-carriers produce the bound quantum systems in the nucleuses (Lutephy, 2020), or similar to the gravity in the galaxies and clusters which they don't obey the Newtonian and Einstein gravities. As argued by Lutephy (2020), the galaxies and clusters are large scale bound quantum systems which the potential is limited to the boundary of galaxies and clusters and then we see very different observations mistakenly concerned to an unobserved thing called dark matter. It is funny that the scientists have calculated the dark matter profiles to illustrate the failure of the Newtonian and Einstein gravities whereas that this is inverse engineering of an imaginary thing.

We have argued here that the change of potential boundary causes to vary the size of gravity but not its formula fundamentally. We have gravity in different systems, not different gravities.

By the way we find that the Newtonian gravity is generalized to the quantum bound systems related to the wavelength of the force carriers or wavelength of the test mass itself as a self-force carrier. Then the gravity in the galaxies and clusters as the large scale quantum bound systems and gravity in the nucleuses as the strong nuclear force and true rainbow's gravity all are identical and unified.

Relativistic Rainbow's gravity scientists believe that the metric components depend to the energy of the test mass but they have not proposed a unified mechanism for refraction and gravity. But here we indicate that the refraction of light is the gravity and even we indicate that the mechanical potential of the light's diffraction is the fundamental gravity. Of course we will see that the size of the gravity is not alone factor for differences in different systems but the fundamental gravity is varied in the vector too and even we see that the fundamental gravity is not directed to the side of the center against the Newtonian gravity suppose it mimics the Einstein general relativity rather than the Newton's gravity. The fundamental gravity is independent so that via assuming some approximations it is transferred to the Newtonian gravity and via other assumptions approximately transferred to the Einstein Gravity in the level of the rainbow's gravity too. Then the fundamental gravity is not indeed a modification for Newtonian and Einstein's gravities but here we return to the fundamental gravity independently and reproduce approximate models hereafter.

These phenomena are generalized also to the light diffraction and light scattering (Lutephy, 2021). In this book we find that the path of photons in the diffraction despite the path derived by Huygens Fresnel principle is not straight but the paths are curved, that is, the paths are quantized however interestingly the quantum mechanical path quantitation coincide the pattern driven by Huygens Fresnel mathematical model. Also we find that absorption of the photons by particles is sourced by fundamental gravity and then the gravity has an essential role in the thermodynamics.

\section{Snell's Law via the Fundamental Gravity}

Here the base of gravity is the light velocity in fully Variable Light Speed (VSL) universe which is derived completely by alliance of the Mach's mechanics and Planck's natural units and generalized Minkowski metric in fully variable light speed universe (Lutephy, 2019a). By this method it is extracted a formula fir light speed variation in each point of the universe and we have:

$c=\frac{\diamond}{\sum_{i} \frac{m_{i}}{r_{i}}}$

where, $c$ is the photon speed and $\mathrm{m}$ is the mass of the particles and $r$ is distance of the particles from the photon and $\diamond$ is a universal constant and we call this equation here the Alpha Genesis Prime. 
This equation is derived by Alliance of the Mach inertia principle and Planck's quantum natural units and generalized Minkowski metric.

Newtonian mechanics is inconsistent with VSL for that it is ever attraction and does not obey exclusively dependency of the light speed to the gravitational potential.

But here we find the fundamental face of the Newtonian mechanics for its generality in N-body problem in fully VSL universe.

The newton's gravity should be reorganized on the Alpha-Genesis-Prime (Eq. 6). This means that the newton's force has a component ever tangent on the light velocity. In $\mathrm{N}$-body problem, the role of tangential component is to justify the variation of light speed via Newtonian type force.

When the light is moving in the space of N-body universe, then the light speed is varied by Alpha Genesis Prime (Eq. 6) and then equivalently we can consider a vector force from $\mathrm{N}$-bodies in which the variation of light speed by them is additionally equal to what the equation of Alpha Genesis Prime does show it.

Then we have ever a tangential component that:

$f_{i \vec{c}}=\frac{G m_{i}}{r_{i}^{2}} \frac{\vec{r} \cdot \vec{c}}{|\vec{r}||\vec{c}|}$

The Alpha Genesis Prime should be confirmed by this force along the path of the light in the N-body space (universe).

By Alpha Genesis Prime (Eq. 6) it is deduced that:

$\Delta c=-\frac{\diamond}{\Theta^{2}} \Delta \Theta$

where we define a parameter called here absolute density $\Theta$ that:

$\Theta=\sum_{i} \frac{m_{i}}{r_{i}}$

We have:

$\Delta \Theta=-\sum_{i} m_{i} \frac{\Delta r_{i}}{r_{i}^{2}}$

Then from Eq. $(8,10)$ we deduce:

$\Delta c=-\frac{\diamond}{\Theta^{2}} \sum_{i} \frac{m_{i} \Delta r_{i}}{r_{i}^{2}}$

We know mathematically that:
$\Delta r_{i}=\Delta r \frac{\vec{r} \cdot \vec{c}}{|\vec{r}||\vec{c}|}$

Then from Eq. $(11,12)$ we obtain:

$\Delta c=-\frac{\oslash}{\Theta^{2}} \sum_{i} \frac{m_{i}}{r_{i}^{2}} \frac{\vec{r} \cdot \vec{c}}{|\vec{r}||\vec{c}|} \Delta r$

Also via the Newton's second law we have:

$\frac{G m_{i}}{r_{i}^{2}} \frac{\vec{r} \cdot \vec{c}}{|\vec{r}||\vec{c}|}=\frac{\Delta_{i} c}{\Delta t}$

where, $\Delta_{i} c$ is the change of the light speed by $\mathrm{i}$-th particle with mass $m_{i}$.

From Eq. (13) and (14) we deduce that:

$\Delta c=-\frac{\diamond}{\Theta^{2} G} \sum_{i} \Delta_{i} c \frac{\Delta_{r}}{\Delta t}$

Substituting $c=\Delta r / \Delta t$ into the above equation yields to:

$\Delta c=-\frac{\diamond c}{\Theta^{2} G} \sum_{i} \Delta_{i} c$

Clearly we have:

$\Delta c=-\sum_{i} \Delta_{i} c$

And then from Eq. $(16,17)$ it is deduced that:

$G=\diamond \frac{c}{\Theta^{2}}$

Substituting Eq. $(6,9)$ in the Eq. (18) we obtain:

$G=\frac{c^{3}}{\diamond}$

Also we obtain that:

$\diamond=\frac{c_{3}}{G}$

And constancy of $c^{3} / G$ has been verified previously via alliance of the generalized Minkowski metric and Planck quantum natural units and Mach's mechanics (Lutephy, 2019a).

Then from Eq. $(6,20)$ for a mass-point affected gravitationally by a mass like the sun with size $M$ in a distance $R$ in two body problem universe we have that: 


$$
c=\frac{c_{0}}{\frac{G_{N}}{c_{0}^{2}} \sum_{i} \frac{m}{r}+\frac{G_{N}}{c_{0}^{0}} \frac{M}{R}}
$$

Then from Machian relation $G_{N} \sum m / r=c^{2}$ we find:

$$
\begin{aligned}
& c=\frac{c_{0}}{1+\frac{G_{N} M}{c_{0}^{2} R}} \\
& c \cong c_{0}\left(1-\frac{2 G_{N} M}{c_{0}^{2} R}\right)
\end{aligned}
$$

And this is identical with the light speed derived in the Schwarzschild metric (1916). Equation (23) is an evidence to indicate that the fundamental gravity is not centrifugal force but when the light is moving radial toward the mass $M$; the speed of the light is decreased despite the Newton's gravity. Of course failure of the Schwarzschild metric is that at the infinity, the light speed is $c_{0}$ whereas that here the fundamental gravity is fully VSL, that is, the light speed is determined by the matter only in the definition of the Mach inertia principle.

By the way from Eq. $(7,20)$ we find:

$$
f_{i \vec{c}}=\frac{m_{j} c^{3}}{\diamond r_{i}^{2}} \frac{\vec{c} \cdot \vec{r}_{i}}{|c|\left|r_{i}\right|}
$$

This is tangential component of the fundamental gravity which its duty is to justify the light speed on the line of the Alpha Genesis Prime.

In the vector calculus, each vector is divided to the tangent and normal (perpendicular) components on the Pythagorean Theorem and then the complete vector of the fundamental gravity is drawn as:

$$
f_{i}=\frac{m_{i} c^{3}}{\diamond r_{i}^{2}}\left[\frac{\vec{r}_{i} \cdot \vec{c}}{\left|\vec{r}_{i}\right||\vec{c}|}-\frac{\vec{r}_{i} \cdot \hat{c}}{\left|\vec{r}_{t}\right||\hat{c}|}\right]
$$

where, $\hat{c}$ is assumed as a vector in the size of the light speed, normal to the light velocity vector $\vec{c}$ so that $\hat{c} \times \vec{c}=c^{2}$.

Equivalently we can write that:

$$
f_{i}=\frac{m_{i} c^{3}}{\nabla r_{i}^{2}}\left[\mid \cos \varphi \frac{\vec{c}}{|\bar{c}|}-\sin \varphi \frac{\hat{c}}{|\hat{c}|}\right]
$$

where, $\varphi$ is the angle between vectors, light velocity $\vec{c}$ and the distance $\vec{r}$.

Or we can write that:
$f_{i}=\frac{G m_{i}}{r_{i}^{2}}[\cos \varphi \vec{M}-\sin \varphi \vec{N}]$

where, $\vec{M}$ is a unit vector tangent to the light velocity and $\vec{N}$ is a unit vector normal to the light velocity. Or we can say that the generalized Minkowski metric while the light speed is fully variable by Alpha Genesis Prime is the fundamental gravity in the language of the metric tensor.

As we see the fundamental force here is not centrifugal for that the sign of the normal component $\vec{N}$ is negative and this negative sign has a very important role. If the sign of the normal component was positive, the force was identical with Newtonian gravity and the light speed was increasing radially by force towards the active mass whereas that according to the Alpha Genesis Prime, the light speed is decreased similar to the Schwarzschild metric. In reality the fundamental gravity seems a Newtonian mode of the Einstein general relativity.

Then negative sign of the normal component is logical result of the Alpha Genesis Prime or generally the result of every kind of VSL which the light speed is increased by distance.

In vector mode, the reestablished Newton law, is the same Newton's law but just the sign of the normal component of the vector $\vec{r}$ on the light velocity is reversed.

According to the Newton's second law and Eq. (27) we obtain:

$\frac{m c^{3}}{r^{2}} \cos \varphi=\frac{d c}{d t}$

$\frac{m c^{3}}{r^{2}} \sin \varphi=\frac{c d \theta}{d t}$

In the $x-y$ coordinate system mathematically we have:

$\varphi=\theta-\operatorname{arctg}(d y / d x)$

Substituting Eq. $(30)$ into the Eq. $(28,29)$ it is deduced that:

$\frac{G m}{r^{2}} \cos (\theta-\operatorname{arctg}(d y / d x))=d c$

$\frac{G m}{r^{2}} \sin (\theta-\operatorname{arctg}(d y / d x))=c d \theta$

Then we deduce that: 
$\frac{d c}{c} \cot (\theta-\operatorname{arctg}(d v / d y)) d \theta$

In the additional form for a material surface we have:

$d y / d x=0$

And then from Eq. $(33,34)$ we deduce that:

$\frac{d c}{c}=\cot (\theta) d \theta$

Integration on the Eq. (35) results:

$\ln c=\ln |\sin \theta|$

Then it is deduced that:

$c \sin \theta=c^{\prime} \sin \theta^{\prime}$

And this is the Snell's law of the light refraction, argued here by fundamental gravity.

For straightforward argument consider a material surface which the light passes it at an angle $\varphi$ from $y$ axis. The vector $\vec{r}$ is ever along the y-axis and then we have that:

$d \varphi=d \theta$

Substituting Eq. $(38)$ in the Eq. $(28,29)$ it is deduced:

$$
|f| \cos \varphi=\frac{d c}{d t}
$$

$|f| \sin \varphi=\frac{c d \varphi}{d t}$

And the solution of this apparatus is clearly the Snell's law (Eq. 37).

Also from fundamental gravity (Eq. 26) we obtain:

$$
\left|f_{i}\right| r_{i}^{2}=\frac{m_{i} c^{3}}{\diamond}
$$

In reality we have

$$
\begin{aligned}
& \frac{m_{i} c^{3}}{\nabla r_{i}^{2}}=\frac{d c}{d t} \\
& \frac{m_{i} c^{3}}{\diamond r_{i}^{2}}=\frac{c^{2} d \varphi}{d l}
\end{aligned}
$$

where, $d l$ is the differential arc of the path.
By the way substituting $d \varphi / d l=1 / R$ into the Eq. (43) we obtain:

$$
\frac{m_{i} c^{3}}{\Delta r_{i}^{2}}=\frac{1}{R}
$$

$$
\frac{m_{i}}{r_{i} \sum_{j} \frac{m}{r}}=\frac{r_{i}}{R_{i}}
$$

This is a version of the fundamental gravity and interestingly we see that the equation is completely non scale.

And as argued in the book "non scale mechanics" (Lutephy, 2019b), the Eq. (45) is deduced from the below fundamental equations that:

$\left|f_{1}\right| r_{1}^{2}=\left|f_{2}\right| r_{2}^{2}=\cdots=\left|f_{n}\right| r_{n}^{2}=c^{3}$

$\left|f_{1}\right| r_{1}+\left|f_{2}\right| r_{2}+\cdots+\left|f_{n}\right| r_{n}=c^{2}$

The Eq. (46) does show that the inverse square law of the Newton is proportional and against the Newton dynamics, there is no a universal constant $G$ that $f r^{2}=G m$ but the correct sentence is relative sentence in the $\mathrm{N}$-body universe that $f r^{2}=f^{\prime} r^{\prime 2}$.

In realty from Eq. $(6,43)$ we have:

$\frac{c^{2} d \varphi}{d l}=\frac{m_{i} c^{2}}{r_{i}^{2} \sum_{i} \frac{m}{r}}$

Then it is deduced that:

$\frac{m_{i} c^{2}}{r_{i}^{2} \Theta}=a$

Or equivalently:

$\frac{G_{N} m_{i}}{r_{i}^{2}}=\frac{G_{N} \sum_{i} \frac{m}{r}}{c^{2}} a$

And this equation is what we see it in the galaxies and clusters versus the fictitious dark matter (Lutephy, 2020).

We can follow on the metric method too. Via generalized Minkowski metric (Lutephy, 2019a) we find that:

$g_{00}=-c^{2}$

In relativistic mode (Lutephy, 2019a) we have: 


$$
c^{2}=\frac{1}{\chi_{2} \sum \frac{m}{r}}
$$

Where:

$$
\chi_{2}=G / c^{4}
$$

Then we have:

$$
g_{00} \sum \frac{m}{r}=-X_{2}^{-1}
$$

From the geodesy equation we have:

$$
\frac{\partial^{2} x_{\mu}}{\partial t^{2}}=\frac{\partial}{\partial x_{\mu}} g_{00}
$$

Substituting, $\Theta=\sum m / r$ in the Eq. (54) and then substituting $g_{00}$ from Eq. (54) in the Eq. (55) it is deduced that:

$$
\begin{aligned}
& \frac{\partial^{2} x_{\mu}}{\partial t^{2}}=\chi_{2}^{-1} \frac{\partial(1 / \Theta)}{\partial x_{\mu}} \\
& \frac{\partial^{2} x_{\mu}}{\partial t^{2}}=\frac{c^{4}}{G^{2} \Theta^{2}} \frac{\partial(G \Theta)}{\partial x_{\mu}} \\
& \frac{\partial^{2} x_{\mu}}{\partial t^{2}}=\frac{\partial \varphi}{\partial x_{\mu}}
\end{aligned}
$$

And the Eq. (56.3) is Newtonian gravity derived by fundamental gravity in metric mode. Of course from equation $\varphi=c^{1}$ and substituting into the Eq. (56.3) we obtain that:

$$
\frac{\partial^{2} x_{\mu}}{\partial t^{2}}=\frac{\partial c^{2}}{\partial x_{\mu}}
$$

Also for geodesy it has been defined that:

$$
\delta \int d s=0
$$

But for light the $d s$ is ever zero and then this equation doesn't work.

For light speed we have:

$$
d t=d l / c
$$

This equation is on the equivalency of the time and light speed and $d t$ has the role of $d s$ while the time is not curved. The time dimension indicates the light speed in each point of the place and independent definition of the time and light speed is one of the mistakes by of the Einstein. If the time is curved then naturally the light speed should be ever constant whereas that in Einstein general relativity, the light speed is variable independently.

By the way when the curvature of the space is fully driven by VSL, we can find that the least path is the path that:

$\delta \int \frac{d l}{c}=0$

And then from Eq. $(58,60)$ it is deduced that:

$$
\delta \int d t=0
$$

And this equation verifies the Fermat's Principe of minimum time. And then the Fermat least time principle does violate the Einstein theorem in his general relativity for the equation of the geodesy that $\delta \int d t=0$.

Interestingly the Fermat's principle of the least time, it results the Snell's law of the light refraction, verifying our results here.

\section{Fundamental Rainbow's Gravity}

Physicists have accepted that the light speed is not varied fundamentally in the light refraction and mainstream scientists have accepted that the light speed variation is virtual, that is, what it is observed as the slowing of the light speed in the refraction is the group velocity. But the gravitational potential as the integral of the masses per distances in fundamental gravity is not ever surrounded on the whole of the observable universe. As it is argued by Lutephy (2020), the gravitational potential in the nucleuses is limited to the wavelength of the force-carriers which naturally defines the range of the force which causes to increase highly the intensity of the gravity as the source of the strong nuclear force.

In fundamental gravity we have a mode which is weakest for that the potential is surrounded on the observable universe and a mode for the gravity which the integration of the potential is not on the whole of the observable universe but in the range of the wavelength of the quantum mechanical force-carriers. Then for a photon itself in the range of the photon's wavelength, photon plays the role of a force-carrier limited to the masses inside a sphere with radius equal to the photon's wavelength.

Thus, we have a system limited to a volume that the photon is itself quantum mechanically gravity-carrier between the particles in the range of the photon's wavelength and then a sphere with radius equal to the wavelength of the photon which photon is sited in the center is a quantum bound system as a quasi-universe 
for rainbow gravity and then the light speed in the light refraction is changed fundamentally.

By the way from Eq. (50) for a photon's gravitational interaction in the range of the wavelength of the photon $r_{i}<\lambda$ we have:

$$
g=\frac{m_{i} c^{2}}{r_{i}^{2} \sum_{\left.i\right|_{i}<\lambda} \frac{m_{i}}{r_{i}}}
$$

This equation is in reality generalization of the strong nuclear force which it has been argued by Lutephy (2020).

Equivalently we can write that:

$$
g=G_{N} \frac{\sum_{i} \frac{m_{i}}{r_{i}}}{\sum_{i \mid r_{i}<\lambda} \frac{m_{i}}{r_{i}}} \times \frac{m_{i}}{r_{i}^{2}}
$$

This is the fundamental equation of the the rainbow's gravity.

Then we have a generalized $G$ in quantum bound system of the photons and generally the particles as:

$$
G=G_{N} \frac{\sum_{i} \frac{m}{r}}{\sum_{i \mid p_{i}<\lambda} \frac{m_{i}}{r_{i}}}
$$

We can see that when the wavelength of the photon is equal to the radius of observable universe, then the rainbow's gravity is at the same $G$, the newton's gravity so that $G=G_{N}$ whereas that how much the wavelength of the photon to be shorter, the $\mathrm{G}$ becomes larger so that for visible photons, the $\mathrm{G}$ becomes inasmuch as large that it curves the light intensely as well as the curvature of the light around the fictitious black holes.

Thus, the fundamental gravity in photonic quantum bound systems is reduced to the true rainbow's gravity. Of course according to the de Broglie wave equation $m v=h / \lambda$, each particle also has relevant quantum mechanical evolution via its wavelength which has been discussed for nucleuses previously (Lutephy, 2020).

By the way the complete equation of the rainbow's gravity is derived by fundamental gravity as:

$$
g=\frac{c^{2}}{\sum_{i \mid r_{i}<\lambda} \frac{m_{i}}{r_{i}}} \times \frac{m_{i}}{r_{i}^{2}}[\cos \varphi \vec{M}-\sin \varphi \vec{N}]
$$

We see that when the wavelength is larger, the rainbow's gravity is weaker in a medium with constant number density. Then energetic photons are refracted much more.
On the Eq. (65) we can calculate the change of the light speed in N-body problem. This is not a regular process and it needs to resolve the apparatus of the equations. But from Eq. (65) approximately we find that Alpha Genesis Prime (Eq. 6) is generalized to the below equation included to a nonlinear term $\gamma_{r<\lambda}$ that:

$c=\frac{\diamond}{\sum_{i} \frac{m}{r}+\gamma_{r<\lambda}}$

Where we have non-scale proportionality as:

$\gamma_{r<\lambda} \sum_{i \in r<\lambda} \frac{m_{i}}{r_{i}}=\gamma_{0} \sum_{i \in r>\lambda} \frac{m_{i}}{r_{i}}$

So that $\gamma_{0}$ is assumed as a constant to justify the equation then in the rainbow's gravity, the linear equation of the Alpha Genesis Prime is not valid longer. Interestingly we find an approximation so that:

$\gamma_{r<\lambda} \approx \sum_{i} \frac{m_{i}}{r_{i}^{\alpha(\lambda)}}$

This equation fulfills both the properties, diminishing the term for $r>\lambda$ and enhanced $G$ for smaller wavelengths and then we have:

$c=\frac{\diamond}{\sum_{i} \frac{m}{r}+\sum_{i} \frac{m_{i}}{r_{i}^{\alpha(\lambda)}}}$

On this nonlinear variable light speed in the rainbow's gravity mode which is derived by fundamental gravity, we can write a metric so that:

$d s^{2}=-c_{0}^{2}\left(1+\frac{G_{N} m}{c_{0}^{2} r^{\alpha(\lambda)}}\right)^{-1} d t^{2}+\left(1+\frac{G_{N} m}{c_{0}^{2} r^{\alpha(\lambda)}}\right) d r^{2}+r^{2} d \Omega^{2}$

In a different method the scientists (Hendi et al., 2016) have extracted a rainbow's metric for spherical symmetric space-time in d-dimensions as:

$d s^{2}=-\frac{\psi(r)}{f(E)^{2}} d t^{2}+\frac{1}{g(E)^{2}}\left[\frac{d r^{2}}{\psi}+r^{2} d \Omega^{2}\right]$

Where according to the (Hendi et al., 2016):

$d \Omega^{2}=d \theta_{1}+\sum_{i=2}^{d-2} \prod_{j=1}^{i-1} \sin ^{2} \theta_{j} d \theta_{i}^{2}$

A solution is Reissner-Nordstrom black hole solution (Hendi et al., 2016) as: 


$$
\psi(r)=1-\frac{m}{r^{d-3}}-\frac{2 A r^{2}}{(d-1)(d-2)}+\frac{2(d-3) q^{2}}{(d-2) r^{2(d-3)}}
$$

The scientists have used d-dimensions to find such solutions to agree with cosmic observations of the gamma bursts. But by comparison of Eq. $(70,71)$, if we assume that:

$$
\psi(r)=\left(1+\frac{G_{N}}{c_{0}^{2}} \frac{m}{r^{\alpha(\lambda)}}\right)
$$

Then we deduce that:

$$
f(E)=g(E)=1
$$

Then in comparison with the equation of Modified Dispersion Relation (MDR) we obtain that:

$$
E^{2}-p^{2}=m^{2}
$$

And we understand that the dependency of the light speed to the photon wavelength doesn't change the energy-momentum (Eq. 76).

We find here that the generalization of the general relativity to the quantum bound systems can be treated on the $\Psi(r)$ only. For example when we assume that $\alpha(\lambda)=3$, than for universe matter we find $\Theta_{U} \equiv 10^{-25}$ and then the effect of the universe potential almost is diminished and it is appeared a high amplitude micro-gravity in general relativity.

Or if we assume that $\alpha(\lambda)=4$, then the metric tensor (Eq. 70) shows the strong nuclear force limited to the nucleuses in general relativity.

Now we find perfect formula of refractive index which it is obtained as follows:

$$
c n=c_{0}
$$

$$
n=\frac{\sum_{i} \frac{m_{i}}{r_{i}}+\gamma_{r<\lambda}}{\sum_{i} \frac{m_{i}}{r_{i}}}
$$

$$
n \cong 1+\frac{G_{N}}{c_{0}^{2}} \sum \frac{m_{i}}{r_{i}^{\alpha(\lambda)}}
$$

We find that the rainbow's gravity is related to a nonlinear potential term which causes to appear dispersion relations in the gravity.

By the way we find here that the refraction is happened even out of the material bar in the range of the wavelength of the incident photons. This means that if a laser beam to pass around a material bar, the refraction will be done. Such an experiment was performed previously by Mahmoud Hessaby and he realized that the laser beams are refracted by material bar and then Mahmoud Hessaby $(1947 ; 1948)$ for correlation of the refractive index to the volume density theorized the theory of the extended particles.

Of course the Hessaby's report for laser beam bending around a material bar mistakenly has been considered as the Gaussian diffraction whereas that the diffraction is symmetric and the Hessaby's report was indeed the light refraction by material bar as we see that it is related to the rainbow's gravity.

Hessaby's mistake in the interpretation of the phenomenon was that he took into account the correlation of refractive index to the volume density whereas that such a correlation is not fundamental. In fact the volume density is not fundamental for reality that the absolute density is not mass embedded in a volume but it is formulized by integral of the masses per distances from the point we want to calculate the absolute density there. The true density in the fundamental gravity is absolute density (potential) and what the scientists have considered as the mass embedded in the volume is an approximation. There is no any volume fulfilled uniformly by matter and simply we find falsification for volume density in the fundamental physics. Each object is included to the discrete particles in a region of the space and definition of the volume density for light traveling between the atoms is meaningless.

We should notice that we don't deny the extended particle theory but we find that the refraction is not by extended particles even if the particles are extended. In fact the quantum mechanics is a kind of extended particle theory. Difference is that in extended-particle theory, the mass is integral of the energy of extended particle on the whole of the space whereas that in the quantum mechanics, the integral of the existence probabilities is at the order of the unity on the whole of the space.

In the Einstein general relativity, the curvature is proportional to the energy tensor and then general relativity requires extended-particle energy which fulfills the continuity of the space. In Einstein general relativity, the space should be fulfilled with the energy and this leads to the extended definition of the energy. Then the continuum and extended-particle theory of the Mahmoud Hessaby is in reality complementary of the Einstein general relativity.

\section{Conclusion}

We find here that the light refraction is in reality the effect of the gravity in bound quantum systems which the potential is limited to the wavelength of the force-carriers and accordingly we argue the Snell's law of the light 
refraction by the gravity. On the other hand we find a metric tensor which mimics the light refraction in the fundamental rainbow's gravity.

\section{Acknowledgement}

We need to thanks from the scientists who have studied along the times in the part of the light refraction in which they have helped us presently to find a solution matched mathematically and experimentally.

\section{Ethics}

This article is original and contains unpublished material. The corresponding author confirms that all of the other authors have read and approved the manuscript and no ethical issues involved.

\section{References}

Ali, A. F., \& Khalil, M. M. (2014). A proposal for testing gravity's rainbow. Europhysics letters. Volume 110, No 2. doi.org/10.1209/0295-5075/110/20009

Ali, A. F., Faizal, M., \& Majumder, B. (2015). Absence of an effective horizon for black holes in gravity's rainbow, Europhys. Lett. 109, 20001. doi.org/10.1209/0295-5075/109/20001

Amelino-Camelia, G., Ellis, J., Mavromatos, N. E., \& Nanopoulos, D. V. (1997). Distance measurement and wave dispersion in a Liouville-string approach to quantum gravity. International Journal of Modern Physics A, 12(03), 607-623.

https://www.worldscientific.com/doi/abs/10.1142/S 0217751 X97000566

Amelino-Camelia, G., Ellis, J., Mavromatos, N. E., Nanopoulos, D. V., \& Sarkar, S. (1998). Tests of quantum gravity from observations of $\gamma$-ray bursts. Nature, 393(6687), 763-765.

https://www.nature.com/articles/31647

Amelino-Camelia, G., Freidel, L., Kowalski-Glikman, J., \& Smolin, L. (2011). Principle of relative locality. Physical Review D, 84(8), 084010.

https://journals.aps.org/prd/abstract/10.1103/PhysRe vD.84.084010

Antoniadis, I., Arkani-Hamed, N., Dimopoulos, S., \& Dvali, G. (1998). New dimensions at a millimeter to a Fermi and superstrings at a TeV. Physics Letters B, 436(3-4), 257-263. doi.org/10.1016/S0370-2693(98)00860-0

Awad, A., Ali, A. F., \& Majumder, B. (2013). Nonsingular rainbow universes J. Cosmol. Astropart. Phys, 10(052), 1308-4343.

Banks, T., \& Fischler, W. (1999). A model for high energy scattering in quantum gravity. arXiv preprint hepth/9906038. https://arxiv.org/abs/hep-th/9906038
Barrow, J. D., \& Magueijo, J. (2013). Intermediate inflation from rainbow gravity. Physical Review D, 88(10), 103525. https://journals.aps.org/prd/abstract/10.1103/Phys RevD.88.103525

Chatrchyan, S., Swanson, J., \& Khachatryan, V. (2012a). CMS Collaboration, Search for dark matter and large extra dimensions in monojet events in ppcollisions at $\sqrt{ }_{s=7 \mathrm{TeV}}$, J. High Energy Phys. 1209(9), 094. doi.org/10.1007/JHEP09(2012)094

Chatrchyan, S., Lehti, S., \& Hindrichs, O. (2012b). CMS Collaboration, Search for microscopic black holes in ppcollisions at $\sqrt{s}_{s}=7 \mathrm{TeV}$, J. High Energy Phys. 1204, 061. Available electronically from https://hdl.handle.net/1969.1/180353

da Rocha, R., \& Coimbra-Araujo, C. H. (2006). Extra dimensions at the CERN LHC via mini-black holes: Effective Kerr-Newman brane-world effects. Physical Review D, 74(5), 055006. https://journals.aps.org/prd/abstract/10.1103/Phys RevD.74.055006

Dimopoulos, S., \& Landsberg, G. (2001). Black holes at the large hadron collider. Physical Review Letters, 87(16), 161602.

https://journals.aps.org/prl/abstract/10.1103/Phys RevLett.87.161602

Emparan, R., Horowitz, G. T., \& Myers, R. C. (2000). Black holes radiate mainly on the brane. Physical Review Letters, 85(3), 499. https://journals.aps.org/prl/abstract/10.1103/PhysRe vLett.85.499

Feng, Z. W., \& Yang, S. Z. (2018). Rainbow gravity corrections to the entropic force. Advances in High Energy Physics, 2018. doi.org/10.1155/2018/5968284

Galán, P., \& Marugán, G. A. M. (2004). Quantum time uncertainty in a gravity's rainbow formalism. Physical Review D, 70(12), 124003. https://journals.aps.org/prd/abstract/10.1103/Phys RevD.70.124003

Garattini, R. (2013). Distorting general relativity: Gravity's rainbow and $\mathrm{f}(\mathrm{R})$ theories at work. Journal of Cosmology and Astroparticle Physics, 2013(06), 017. https://iopscience.iop.org/article/10.1088/14757516/2013/06/017/meta

Garattini, R., \& Majumder, B. (2014a). Naked singularities are not singular in distorted gravity. Nuclear Physics B, 884, 125-141. doi.org/10.1016/j.nuclphysb.2014.04.014

Garattini, R., \& Majumder, B. (2014b). Electric charges and magnetic monopoles in Gravity's Rainbow. Nuclear Physics B, 883, 598-614. doi.org/10.1016/j.nuclphysb.2014.04.005

Garattini, R., \& Mandanici, G. (2012). Particle propagation and effective space-time in Gravity's Rainbow. Physical Review D, 85(2), 023507. https://journals.aps.org/prd/abstract/10.1103/PhysRe vD.85.023507 
Giddings, S. B., \& Thomas, S. (2002). High energy colliders as black hole factories: The end of short distance physics. Physical Review D, 65(5), 056010. https://journals.aps.org/prd/abstract/10.1103/PhysRe vD.65.056010

Gim, Y., \& Kim, W. (2014). Thermodynamic phase transition in the rainbow Schwarzschild black hole. Journal of Cosmology and Astroparticle Physics, 2014(10), 003. https://iopscience.iop.org/article/10.1088/14757516/2014/10/003/meta

Hackett, J. (2006). Asymptotic flatness in rainbow gravity. Classical and Quantum Gravity, 23(11), 3833. https://iopscience.iop.org/article/10.1088/02649381/23/11/010/meta

Hendi, S. H., Eslam Panah, B., Panahiyan, S., \& Momennia, M. (2016). Gravity's Rainbow and Its Einstein Counterpart. Advances in High Energy Physics, 2016. doi.org/10.1155/2016/9813582

Hessaby, M. (1947). “continuous particles”. Proceedings of the national academy of scineces of the united states of America. 33 (6): 189-194.

Hessaby, M. (1948). Theoretical evidence for the existence of a light-charged particle of mass greater than that of the electron. Physical Review, 73(9), 1128. https://journals.aps.org/pr/abstract/10.1103/PhysRev .73 .1128

Leiva, C., Saavedra, J., \& Villanueva, J. (2009). Geodesic structure of the Schwarzschild black hole in rainbow gravity. Modern Physics Letters A, 24(18), 1443-1451. https://www.worldscientific.com/doi/abs/10.1142/S 0217732309029983

Li, H., Ling, Y., \& Han, X. (2009). Modified (A) dS Schwarzschild black holes in Rainbow spacetime. Classical and Quantum Gravity, 26(6), 065004. https://iopscience.iop.org/article/10.1088/02649381/26/6/065004/meta
Liu, C. Z., \& Zhu, J. Y. (2008). Hawking radiation and black hole entropy in a gravity's rainbow. General Relativity and Gravitation, 40(9), 1899-1911. https://link.springer.com/article/10.1007/s10714008-0607-7

Lutephy, M. (2019a). Explosion of the Light: Alliance of the Planck and Mach and Einstein-Riemannian Physics. idea, 36. doi.org/10.3844/pisp.2019.8.23

Lutephy, M. (2019b). Absolute physics: Non scale mechanics. $1^{\text {st }}$ Ed. Kindle Direct Publishing. ISIN: 1492164216

Lutephy, M. (2020). Explosion of the Science Modification of Newtonian Dynamics Via Mach's Inertia Principle and Generalization in Gravitational Quantum Bound Systems and Finite Range of the Gravity-Carriers, Consistent Merely On the Bosons and the Fermions. Physics International, 11(1), 4-35. doi.org/10.3844/pisp.2020.4.35

Lutephy, M. (2021). Boom!: Fundamental gravity as the potential source of the light refraction and diffraction and scattering and absorption. www.amazon.com. ISBN-10: 979-8721596339.

Meade, P., \& Randall, L. (2008). Black holes and quantum gravity at the LHC. Journal of High Energy Physics, 2008(05), 003.

https://iopscience.iop.org/article/10.1088/11266708/2008/05/003/meta

Pais, A. (2005). Subtle is the Lord: The Science and the Life of Albert Einstein. Oxford: OUP.p. 283. ISBN10: 978-0-19-280672-7.

Schwarzschild, K. (1916). Über das gravitationsfeld eines massenpunktes nach der einsteinschen theorie. Sitzungsberichte der Königlich Preußischen Akademie der Wissenschaften (Berlin, 189-196. https://ui.adsabs.harvard.edu/abs/1916SPAW.......18 9S/abstract 